\title{
Antimicrobial Resistance Patterns of Bacterial Isolates from Blood Culture among HIV/AIDS Patients at Felege Hiwot Referral Hospital, Northwest Ethiopia
}

\author{
Mohabaw Jemal $\left(\mathbb{D},{ }^{1}\right.$ Teshiwal Deress $\left(\mathbb{D},{ }^{2}\right.$ Teshome Belachew ${ }^{D},{ }^{1}$ and Yesuf Adem $(\mathbb{D})^{3}$ \\ ${ }^{1}$ University of Gondar, College of Medicine and Health Sciences, School of Biomedical and Laboratory Sciences, \\ Department of Medical Microbiology, Gondar, Ethiopia \\ ${ }^{2}$ University of Gondar, College of Medicine and Health Sciences, School of Biomedical and Laboratory Sciences, \\ Unit of Quality Assurance and Laboratory Management, Gondar, Ethiopia \\ ${ }^{3}$ Bahir Dar University, College of Medicine and Health Sciences, School of Health Sciences, \\ Department of Medical Laboratory Sciences, Unit of Medical Microbiology, Bahir Dar, Ethiopia
}

Correspondence should be addressed to Mohabaw Jemal; mohabawjemal@gmail.com

Received 20 June 2020; Revised 26 August 2020; Accepted 5 October 2020; Published 20 October 2020

Academic Editor: Luigi Santacroce

Copyright (c) 2020 Mohabaw Jemal et al. This is an open access article distributed under the Creative Commons Attribution License, which permits unrestricted use, distribution, and reproduction in any medium, provided the original work is properly cited.

\begin{abstract}
Background. The emergence and spread of antimicrobial resistance in bacteria is recognized as a global public health problem. Bloodstream infection with antimicrobial-resistant bacteria in HIV/AIDS patients makes the problem more challenging. So, regular and periodic diagnosis and use of the appropriate antimicrobial susceptibility pattern determination is the only option for decreasing the prevalence and development of drug-resistant bacteria. Methods. An institution-based cross-sectional study was conducted among 384 HIV/AIDS patients. Sociodemographic data of patients were recorded using structured questionnaires. Blood cultures were collected with BACTEC aerobic blood culture bottles. A pair of samples was collected from each patient aseptically and incubated at $37^{\circ}$. If samples are positive for bacterial agents, they were subcultured to solid media such as blood agar plate, chocolate agar plate, and MacConkey agar plates. Identification was performed using colony characteristics and standard biochemical techniques. The antimicrobial susceptibility test was determined by the Kirby-Bauer disc diffusion method. Data entry and analysis were performed while using SPSS version 20. Descriptive statistics were performed to calculate frequencies. Results. Altogether, 384 patients were included, and 123 blood cultures were positive, so that the yield was thus $32 \%$. About 46 (37.4\%) of Gram-negative and 77 (62.6\%) of Gram-positive bacterial species were identified. Among Gram-negative bacterial isolates, K. pneumoniae was the leading pathogen, 19 (41.3\%), whereas S. aureus, 38 (49.4\%), was predominant among Gram-positive isolates. In his study, the majority of Gram-positive isolates showed high level of resistance to penicillin, 72 (95.5\%), tetracycline, 55 (71.4\%), and cotrimoxazole, 45 (58.4\%). About 28 (73.6\%) of S. aureus isolates were also methicillinresistant. Gram-negative bacterial isolates also showed a high resistance to ampicillin (91.3\%), tetracycline (91.3\%), and gentamicin (47.8\%). Overall, about 78\% of multidrug resistance was observed. Conclusion. Several pathogens were resistant to greater than five antimicrobial agents, so that proper management of patients with bacteremia is needed, and a careful selection of effective antibiotics should be practiced.
\end{abstract}

\section{Background}

Bacterial bloodstream infections constitute a significant public health problem and present an important cause of morbidity and mortality in HIV-infected patients. Even if it is worldwide, the highest burden is documented in subSaharan countries including Ethiopia [1]. Bacteremia is one of the major leading causes of deaths in HIV-infected patients as compared to without HIV [2]. Conditions such as defective cell-mediated immunity, altered B cell function, 
and defect of neutrophil are leading factors that enhance susceptibility of patients to bacterial infections [3]. Sepsis is another episode of disease among HIV/AIDS and remains the second leading cause of death with an estimated mortality rate of $7 \%$ in the pediatric population [2]. Most of these deaths were reported from facilities in sub-Saharan Africa and Asia where healthcare access, infrastructure, and staffing remains suboptimal. Both Gram-negative and Gram-positive bacterial agents can play a pivotal role in causing high morbidity of blood stream infections. For instance, a survey among HIV-I patients in Malawi showed that 30\% had bloodstream infections, and organisms isolated in this study were mainly $S$. pneumoniae, $S$. aureus, $E$. coli, K. pneumoniae, and M. tuberculosis [2].

Antimicrobial resistance (AMR) is an emerging and serious public health problem among HIV/AIDS patients in both developed and developing countries, though the burden is high in sub-Saharan countries [4-6]. Different studies show that antimicrobial resistance is alarmingly increasing. Mutation and selective pressure by antibiotics during treatment are among documented factors for antimicrobial resistance $[7,8]$. Rising antibiotic resistance rates among E. coli (with resistance to third generation cephalosporins and fluoroquinolones) is particularly problematic, since cephalosporins are the mainstay of empiric therapy for both community-acquired and hospital-acquired bloodstream infections in resource-limited settings. The estimated prevalence of extended spectrum beta-lactamase- (ESBL-) producing Enterobacteriaceae in Asia and sub-Saharan Africa is between $60 \%$ and $90 \%$ [9], highlighting the growing challenge of treating bloodstream infections in these countries [10]. In developing countries, controlling AMR is still challenging because of lack of surveillance systems, poor infection control measures, and self-prescription of antimicrobial agents [11]. An infection with multidrug-resistant bacteria also consumes more healthcare resources [11, 12]. Therefore, this study aimed to assess the antimicrobial resistance patterns of bacterial isolates from blood culture among HIV/AIDS patients at Felege Hiwot Referral Hospital and to provide updated information to regulatory bodies and those who would like to use the findings for the development of intervention strategies as appropriate.

\section{Materials and Methods}

2.1. Study Setting, Design, Population, and Sampling Techniques. An institution-based cross-sectional study was conducted at Felege Hiwot Comprehensive Specialized Hospital, Bahir Dar, Amhara regional state. It was performed to determine antimicrobial resistance patterns of bacterial isolates among bloodstream-suspected HIV/AIDS patients attending ART clinic from July 11 to December 11, 2018. Bahir Dar city is located $576 \mathrm{~km}$ from the capital city of the country, Addis Ababa. Based on the 2007 Census conducted by the Central Statistical Agency (Ethiopia), this city has a total population of 221,990 [13]. The hospital was purposely selected because it is one of the biggest referral hospitals in the region. Currently, it provides inpatient and outpatient services for more than 7 million people in its catchment area and nearby regions. The hospital has inpatient wards, outpatient department, and laboratory service with a total of 400 beds and 9 operating tables. Besides, it has an ART clinic with separate ART Laboratory and ART Pharmacy that serve about 17,569 HIV patients [14]. The sample size (384) was determined by using a single population proportion formula while considering the prevalence of $50 \%$, with a $95 \%$ confidence interval, and a $5 \%$ margin of error. Study participants were recruited by the convenient sampling technique. HIV/AIDS patients suspected of bloodstream infection were included in this study. However, patients who took antibiotics two weeks before data collection and at the time of the study were excluded. In this study, all patients visiting Felege Hiwot Comprehensive and Specialized Hospital for a certain clinical disease diagnosis were considered as a source population. Whereas HIV/AIDS patients who were visiting the ART clinic for bloodstream infection diagnosis during the study period were considered as a target population.

2.2. Data Collection Methods. A pretested, structured questionnaire was prepared from reviewed literature and used to collect sociodemographic information. It was prepared in English, translated to the local language (Amharic), and then translated back into English to check its consistency. Clinical signs such as axillary temperature $\geq 38.5^{\circ} \mathrm{C}$ or $\leq 36.5^{\circ} \mathrm{C}$, pulse $\geq 90^{\circ}$ beats/minute, and frequency of respiration $\geq 20$ minute were used as criteria to suspect bloodstream infection and recruit patients for this study.

\subsection{Laboratory Methods}

2.3.1. Blood Sample Collection. Venous blood samples, $20 \mathrm{~mL}$ (adult) and $2-5 \mathrm{ml}$ (children), were taken from each patient, and $10 \mathrm{~mL}$ (adult) and $2-5 \mathrm{~mL}$ (children) were inoculated into a pair of bottles (Becton, Dickinson, USA) under strict aseptic procedures [12].

2.3.2. Culture and Identification. The blood samples were subsequently incubated for 7 days according to the standards of the World Health Organization (WHO) [15]. The presence of bacteria was indicated when there was evidence of visible microbial growth, hemolysis, turbidity, gas production, and/or coagulation of the broth. About $0.1 \mathrm{ml}$ of the sample was drawn using a sterile syringe from culture bottles showing at least one of the abovementioned indications and plated out on MacConkey, blood, and chocolate agar plates using the streak plate technique. The plates were then incubated at $37^{\circ} \mathrm{C}$ for $18-24$ hours and observed for bacterial growth. The chocolate agar plates were incubated under microaerophilic conditions in a jar for the possible isolation of microaerophiles. Colonies appearing on the agar plates were subcultured for purity, and a minimum of three colonies with identical morphology was selected individually and subjected to identification by standard biochemical tests. Gram-negative bacteria were identified by the oxidase test, indole production, citrate utilization, motility, urease, 
oxidase, gas production, hydrogen sulfide production, carbohydrate fermentation, and lysine decarboxylases production tests [12]. Similarly, Gram-positive bacterial isolates were mainly identified using coagulase and catalase tests. Further differentiation of Streptococcus species was performed by bacitracin/optochin tests. The identification of Haemophilus species was performed using the XV factorsupplemented chocolate agar plate and blood agar plate. Samples were recorded as negative when no growth was detected within seven days' incubation [12].

2.3.3. Antimicrobial Susceptibility Test. Antimicrobial susceptibility tests were carried out using the Kirby-Bauer disc diffusion method as per the Clinical Laboratory Standards Institute (CLSI) guidelines on Muller-Hinton agar [16]. The suspension of 3-5 pure colonies of freshly grown test organisms was prepared equivalent to 0.5 McFarland standards. The surface of Muller-Hinton agar was then completely covered by rotating the swab with the suspension. For fastidious microorganisms, Muller-Hinton agar supplemented with $5 \%$ lysed/defibrinated whole blood was used [17]. The plates were allowed to dry for 3-5 minutes; then, discs were evenly distributed with $24 \mathrm{~mm}$ apart on the inoculated plate using sterile forceps and incubated at $37^{\circ} \mathrm{C}$ for 18-24 hours. The diameter of the zone of inhibition around the disc was measured using a ruler. Results were interpreted as sensitive, intermediate, and resistance based on the CLSI 2016 guideline [16].

The following routinely used antimicrobials were tested: ampicillin $(10 \mu \mathrm{g})$, amoxicillin-clavulanic acid $(30 \mu \mathrm{g})$, ceftriaxone $(30 \mu \mathrm{g})$, ciprofloxacin $(5 \mu \mathrm{g})$, chloramphenicol $(30 \mu \mathrm{g})$, sulfamethoxazole/trimethoprim $(25 \mu \mathrm{g})$, gentamicin $(10 \mu \mathrm{g})$, piperacillin $(100 \mu \mathrm{g})$, tetracycline $(30 \mu \mathrm{g})$, penicillin (10 IU), vancomycin $(30 \mu \mathrm{g})$, erythromycin $(15 \mu \mathrm{g})$, oxacillin $(1 \mu \mathrm{g})$, clindamycin $(2 \mu \mathrm{g})$, and cefoxitin $(30 \mu \mathrm{g})$. Cefoxitin disc $(30 \mu \mathrm{g})$ was used for the detection of MRSA and MR-CoNS. Culture media were incubated at $37^{\circ} \mathrm{C}$ for 16 to 18 hours for MRSA and 24 hours for coagulase-negative Staphylococci (CoNS). Sensitivity results for MRSA and MR-CoNS were reported when the zone of diameter is $\leq 21 \mathrm{~mm}$ and $\leq 24 \mathrm{~mm}$, respectively [16]. Multidrug resistance was defined as the resistance of an isolate to three or more antimicrobial classes tested [18].

2.3.4. Quality Control. Standard reference strains of E. coli (ATCC-25922), S. aureus (ATCC-25923), P. aeruginosa (ATCC-27853), E. faecalis (ATCC 29212), S. pneumoniae (ATCC 49619), and S. pyogenes (ATCC 19615) were used as a media quality control throughout the study.

2.4. Data Analysis and Interpretation. Collected data were entered into Epi-data 3.1 and exported to the SPSS version 20 statistical software for analysis. During analysis, descriptive statistics including mean, frequency, and percentage were used to summarize the data as appropriate. Then, the findings of this study were presented in the form of texts, tables, and graphs as appropriate. A $P$ value of $<0.05$ was considered as statistically significant.

2.5. Ethical Consideration. An official ethical letter (protocol number: DRERC/182/15/MLS) was obtained from the Departmental Research and Ethics Review Committee of Addis Ababa University, Department of Medical Laboratory Sciences. Then, consent was obtained from the study participants after informing their involvement was based on a voluntary basis. Participants who were unwilling to involve in the study and those who need to quit their participation at any stage were informed to do so without any restriction.

\section{Results}

3.1. Demographic Characteristics. A total of 384 bacteremia suspected patients were included in this study. Of these, 157 (41\%) were males and 227 (59\%) were females. They were aged 1-75 years with a mean age of 36 years $(\mathrm{SD} \pm 11.8)$. Distribution according to the age range showed that the majority of the HIV-infected patients were in the age group of $25-44$ years, 252 (65.6\%), followed by those whose age ranged between 45 years and 64 years, 83 (21.8\%), and the least number was between the age group of 15 years and 24 years, 20 (5.3\%) (Table 1). About 123 (32\%) samples were culture-positive, with all of them showing a monobacterial growth, yielding a total of 123 bacterial isolates. A higher positivity rate was seen in children as compared to the adults (Table 1).

3.2. Bacterial Isolates. The bacterial profile from the blood culture of HIV-infected patients revealed that Gram-positive bacterial isolates had the highest percentage of $62.6 \%$ followed by Gram-negatives with 37.4\%. Among Gram-positives, $S$. aureus, $49.4 \%$ (38/77), was the most predominant followed by CoNS, 35\% (27/77), whereas K. pneumoniae was the leading pathogen, $41.3 \%$ (19/46), among Gram-negatives (Figure 1).

3.3. Antibiotic Susceptibility Profile of Gram-Positive Bacterial Isolates. S. aureus isolates exhibited the highest percentage resistance to penicillin, tetracycline, and cotrimoxazole with 36 (95\%), 27 (71\%), and 25 (65\%), respectively (Table 2). Besides, 28 (73.7\%) S. aureus and 18 (70\%) CoNS were methicillin-resistant (Table 3). Among the total number of MRSA isolates, $25 \%$ (7/28) were healthcare-associated, while 21/28 (75\%) were community-associated. About 11/18 (61.1\%) of MR-CoNS isolates were healthcare associated and 7/18 (38.9\%) were community associated (Table 3).

3.4. Antibiotic Susceptibility Profile of Gram-Negative Bacterial Isolates. K. pneumoniae showed a significant level of resistance to tetracycline, 18 (95\%), and chloramphenicol, 17 (89\%). E. aerogenes also showed the highest percentage of resistance to ampicillin, 15 (93.7\%), and tetracycline, 13 (81.3\%) (Table 4). 
TABLE 1: Demographical characteristics of study participants with bacterial bloodstream infection among HIV/AIDS patients in Felege Hiwot Referral Hospital.

\begin{tabular}{|c|c|c|c|}
\hline \multirow{2}{*}{ Characteristic } & \multicolumn{2}{|c|}{ Bacterial status } & \multirow{2}{*}{ Total, $N(\%)$} \\
\hline & Positive, $N(\%)$ & Negative, $N(\%)$ & \\
\hline \multicolumn{4}{|l|}{ Sex } \\
\hline Male & $50(31.8)$ & $107(68)$ & $157(41)$ \\
\hline Female & $73(32.1)$ & $154(67.8)$ & $227(59)$ \\
\hline \multicolumn{4}{|l|}{ Age } \\
\hline $1-14$ & $14(53.8)$ & $12(46.2)$ & $26(6.8)$ \\
\hline $15-24$ & $5(25)$ & $15(75)$ & $20(5.3)$ \\
\hline $25-44$ & $81(32.1)$ & $171(67.8)$ & $252(65.6)$ \\
\hline $45-64$ & $23(27.7)$ & $60(72.3)$ & $83(21.8)$ \\
\hline$>65$ & $0(0)$ & $3(100)$ & $3(0.8)$ \\
\hline \multicolumn{4}{|l|}{ Marital status } \\
\hline Unmarried & $20(36.2)$ & $45(63.8)$ & $65(24.5)$ \\
\hline Married & $57(29.8)$ & $135(70.2)$ & $191(49.7)$ \\
\hline Widow & $3(27.3)$ & $8(72.7)$ & $11(2.9)$ \\
\hline Divorced & $73(33)$ & $43(67)$ & $88(22.9)$ \\
\hline \multicolumn{4}{|c|}{ Educational level } \\
\hline Illiterate & $39(30.7)$ & $72(69.3)$ & $104(27.3)$ \\
\hline Literate & $90(32.6)$ & $186(67.4)$ & $276(72.7)$ \\
\hline \multicolumn{4}{|l|}{ Occupational } \\
\hline Farmer & $11(29.7)$ & $26(70.3)$ & $37(9.6)$ \\
\hline Student & $16(32)$ & $34(68)$ & $50(13)$ \\
\hline House wife & $20(39.2)$ & $32(61.5)$ & $51(13.2)$ \\
\hline Employer & $61(30.6)$ & $156(71.9)$ & $236(61.5)$ \\
\hline Other & $15(53.6)$ & $13(46.4)$ & $10(2.6)$ \\
\hline Total & $123(32)$ & $261(68)$ & $384(100)$ \\
\hline
\end{tabular}

Note. A, government and self-employed; b, jobless and children under 4 years. Age is categorized based on the WHO guidelines.

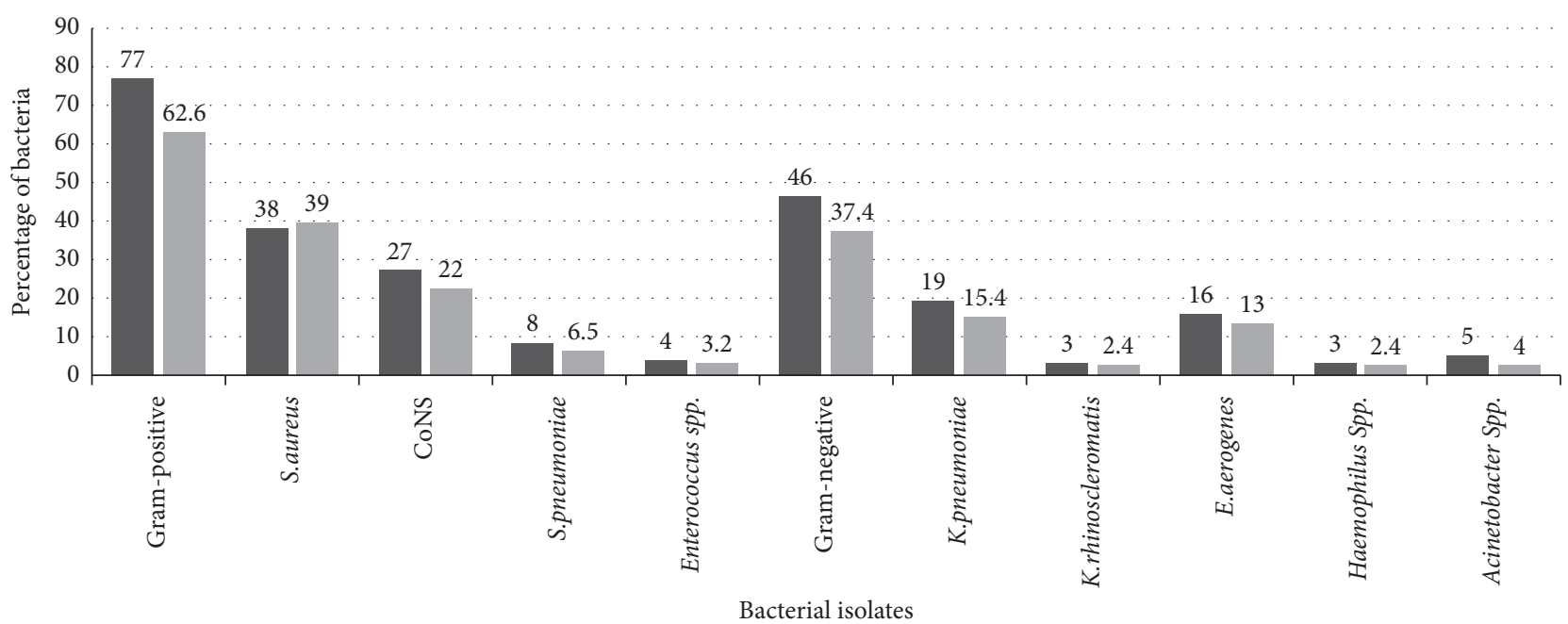

Frequency $(N)$

(\%) of total isolates $N(123)$

FIgURE 1: Distribution of bacterial isolates among blood culture-positive HIV/AIDS patient at Felege Hiwot Referral Hospital.

Regarding multidrug-resistance patterns of bacterial isolates, about $78 \%(96 / 123)$ of them showed multidrug resistant (MDR). Even about $50(40.7 \%)$ of the isolates were resistant to at least five classes of antimicrobial agents (Table 5). The most common MDR Gram-positive bacterial isolates were S. aureus, 48\% (37/77), CoNS, $28.6 \%(22 / 77)$, and K. pneumoniae, $23.9 \%$ (11/46) (Table 5). 
TABle 2: Antibiotic resistance patterns of Gram-positive bacterial isolates among HIV/AIDS-infected patients at Felege Hiwot Referral Hospital.

\begin{tabular}{|c|c|c|c|c|c|c|c|c|c|c|}
\hline \multirow[b]{2}{*}{ Gram-positive } & \multicolumn{10}{|c|}{ Antibiotic resistance profile } \\
\hline & $\begin{array}{c}\text { CIP, } \\
N(\%)\end{array}$ & $\begin{array}{l}\text { COT, } \\
N(\%)\end{array}$ & $\begin{array}{c}\text { CLI, } \\
N(\%)\end{array}$ & $\begin{array}{c}\mathrm{E} \\
N(\%)\end{array}$ & $\begin{array}{l}\text { OXA, } \\
N(\%)\end{array}$ & $\begin{array}{c}\text { FOX, } \\
N(\%)\end{array}$ & $\begin{array}{c}\text { VA, } \\
N(\%)\end{array}$ & $\begin{array}{c}\mathrm{TE}, \\
N(\%)\end{array}$ & $\begin{array}{c}P, \\
N(\%)\end{array}$ & $\begin{array}{c}C, \\
N(\%)\end{array}$ \\
\hline \multicolumn{11}{|l|}{ S. aureus (38) } \\
\hline$S$ & $36(95)$ & $12(32)$ & $27(71)$ & $12(31.5)$ & $9(24)$ & $10(26.3)$ & - & $9(23.6)$ & $2(5.2)$ & - \\
\hline$I$ & $1(2.5)$ & $1(2.6)$ & $0(0)$ & $4(10.5)$ & - & - & - & $2(5.2)$ & - & - \\
\hline$R$ & $1(2.5)$ & $25(65)$ & $11(29)$ & $22(58)$ & $28(73.6)$ & $28(73.7)$ & - & $27(71)$ & $36(95)$ & - \\
\hline \multicolumn{11}{|l|}{ CoNS (27) } \\
\hline$S$ & $25(92.5)$ & $11(41)$ & $19(70)$ & $10(37)$ & $8(30)$ & $9(33.3)$ & - & $7(26)$ & $2(7.4)$ & - \\
\hline$I$ & $0(0)$ & $2(7.4)$ & $0(0)$ & $3(11)$ & - & - & - & $1(3.7)$ & - & - \\
\hline$R$ & $2(7.5)$ & $14(52)$ & $8(30)$ & $14(52)$ & $19(70)$ & $18(66.7)$ & - & $19(70.3)$ & $25(93)$ & - \\
\hline \multicolumn{11}{|c|}{ Enterococcus spp. (4) } \\
\hline$S$ & $4(100)$ & $2(50)$ & - & $3(75)$ & - & - & $4(100)$ & $0(0)$ & $0(0)$ & $4(100)$ \\
\hline$I$ & $0(0)$ & $0(0)$ & - & $0(0)$ & - & - & $0(0)$ & $1(25)$ & $0(0)$ & $0(0)$ \\
\hline$R$ & $0(0)$ & $2(50)$ & - & $1(25)$ & - & - & $0(0)$ & $3(75)$ & $4(100)$ & $0(0)$ \\
\hline \multicolumn{11}{|c|}{ S. pneumoniae (8) } \\
\hline$S$ & $7(87.5)$ & $4(50)$ & - & $5(62.5)$ & - & - & $8(100)$ & $2(25)$ & $1(12.5)$ & $5(62.5)$ \\
\hline$I$ & $0(0)$ & $0(0)$ & - & $0(0)$ & - & - & $0(0)$ & $0(0)$ & $0(0)$ & $3(37.5)$ \\
\hline$R$ & $1(12.5)$ & $4(50)$ & - & $3(37.5)$ & - & - & $0(0)$ & $6(75)$ & $7(87.5)$ & $0(0)$ \\
\hline \multicolumn{11}{|l|}{ Total $(n=77)$} \\
\hline$S$ & $72(93.5)$ & $29(37.6)$ & - & $30(39)$ & - & - & $69(90)$ & $18(23.4)$ & $5(6.5)$ & - \\
\hline$I$ & $1(1.3)$ & $3(3.9)$ & - & $7(9)$ & - & - & $2(2.6)$ & $4(5.2)$ & $0(0)$ & - \\
\hline$R$ & $4(5.2)$ & $45(58.4)$ & - & $40(52)$ & - & - & $6(7.7)$ & $55(71.4)$ & $72(95.5)$ & - \\
\hline
\end{tabular}

Note. CIP, ciprofloxacin; COT, cotrimoxazole; $E$, erythromycin; $P$, penicillin; VA, vancomycin; OXA, oxacillin; CLI, clindamycin; FOX, cefoxitin; CN, gentamycin; TE, tetracycline; $S$, sensitivity, I, intermediate; $R$, resistance; CONS, coagulase-negative Staphylococci.

Table 3: Methicillin-resistant Staphylococci among HIV/AIDS patients at Felege Hiwot Referral Hospital.

\begin{tabular}{lcc}
\hline MR Staphylococci & & Place of acquisition \\
& HCA BSIs, $N(\%)$ & CA BSIs, $N(\%)$ \\
\hline MRSA $(n=28)$ & $7(25)$ & $21(75)$ \\
MR-CoNS $(n=18)$ & $11(61.1)$ & $7(38.9)$ \\
Total $(n=46)$ & $18(39.1)$ & $27(59.8)$ \\
\hline
\end{tabular}

Note. MRSA, methicillin-resistant S. aureus, MR-CoNS, methicillin-resistant coagulase-negative Staphylococci, HCA BSIs, healthcare-associated bloodstream infections, CA BSIs, community-associated bloodstream infections.

\section{Discussion}

Bloodstream bacterial infection is one of the most common infections among HIV-infected individuals [19]. In this study, the overall prevalence of bloodstream infections among HIV/AIDS patients attending the Felege Hiwot Referral Hospital was 32\%. This prevalence is higher than the findings reported in Nigeria, which was $22.9 \%$ [3]. However, our result is comparable with the results reported at Gondar Teaching Referral Hospital, which was 31\% [17]. On the contrary, our result is much lower than the findings reported in China [20] and the USA [21], which is $40 \%$ and $80 \%$, respectively.

The antibiotic susceptibility test results on the current study showed that $40.7 \%$ of the bacterial isolates were resistant to more than five antimicrobial agents. This percentage was significantly higher than the previous finding documented in Gondar, Ethiopia, and 29\% of bacterial isolates were resistant to greater than five antimicrobial agents [17]. The reason for the higher resistance in the current study might be due to the inappropriate use of broad-spectrum antibiotics. This study also illustrated that $58.4 \%$ of Gram-positive and $43.4 \%$ of Gram-negative bacterial isolates were resistant to cotrimoxazole [17]. Although it is very difficult to generalize with this sample size, this study showed the highest resistant profile of many bacterial isolates to cotrimoxazole. Therefore, a large-scale comparative study in the future will answer this critical question. However, this percentage was much lower than results reported in Gondar, Ethiopia, in which $65.3 \%$ of the Grampositive and $100 \%$ of the Gram-negative bacterial isolates were resistant to cotrimoxazole [17]. Another study from Tanzania also showed that $81.3 \%$ Gram-positive and $75 \%$ of Gram-negative bacterial isolates were resistant to cotrimoxazole [22].

In the current study, more than $93 \%$ of Gram-positive and Gram-negative bacterial isolates showed higher sensitivity to ciprofloxacin. More interestingly, all Enterococcus species and S. pneumoniae species were $100 \%$ sensitive to vancomycin (Table 2). On another hand, $98 \%$ and $93.5 \%$ of Gram-negative bacteria showed good sensitivity to ceftriaxone and chloramphenicol, respectively (Table 3). This 
TABle 4: Antibiotic resistance pattern of Gram-negative bacterial isolates among HIV/AIDS patients at Felege Hiwot Referral Hospital.

\begin{tabular}{|c|c|c|c|c|c|c|c|c|c|}
\hline \multirow[b]{2}{*}{ Gram-negative } & \multicolumn{9}{|c|}{ Antibiotic susceptibility pattern } \\
\hline & $\begin{array}{c}\text { CIP, } \\
N(\%)\end{array}$ & $\begin{array}{l}\text { COT, } \\
N(\%)\end{array}$ & $\begin{array}{l}\text { AMP, } \\
N(\%)\end{array}$ & $\begin{array}{l}\mathrm{AMC}, \\
N(\%)\end{array}$ & $\begin{array}{c}\text { CRO, } \\
N(\%)\end{array}$ & $\begin{array}{c}\mathrm{CN} \\
N(\%)\end{array}$ & $\begin{array}{c}\text { TE, } \\
N(\%)\end{array}$ & $\begin{array}{c}\mathrm{C}, \\
N(\%) \\
\end{array}$ & $\begin{array}{c}\mathrm{PI}, \\
N(\%)\end{array}$ \\
\hline \multicolumn{10}{|c|}{ K. pneumoniae $(n=19)$} \\
\hline S & $19(100)$ & $12(63)$ & $2(10.5)$ & $14(73.6)$ & $19(100)$ & $8(42)$ & $1(5)$ & $16(84.2)$ & - \\
\hline$I$ & $0(0)$ & $0(0)$ & $0(0)$ & $1(5.3)$ & $0(0)$ & $1(5.3)$ & $0(0)$ & $0(0)$ & - \\
\hline$R$ & $0(0)$ & $7(37)$ & $17(89)$ & $4(21)$ & $0(0)$ & $10(53)$ & $18(95)$ & $3(16)$ & - \\
\hline \multicolumn{10}{|c|}{ K. rhinoscleromatis $(n=3)$} \\
\hline$S$ & $2(67)$ & $2(67)$ & $1(33.3)$ & $2(66.7)$ & $3(100)$ & $3(100)$ & $0(0)$ & $3(100)$ & - \\
\hline$R$ & $1(33)$ & $1(33)$ & $2(66.7)$ & $1(33.3)$ & $0(0)$ & $0(0)$ & $3(100)$ & $0(0)$ & - \\
\hline \multicolumn{10}{|c|}{ E. aerogenes $(n=16)$} \\
\hline$S$ & $14(87.5)$ & $6(37.5)$ & $1(6.3)$ & $10(62.5)$ & $15(93.7)$ & $6(37.5)$ & $1(6.25)$ & $16(100)$ & - \\
\hline$I$ & $0(0)$ & $0(0)$ & $0(0)$ & $0(0)$ & $0(0)$ & $1(6.2)$ & $2(12.5)$ & $0(0)$ & - \\
\hline$R$ & $2(12.5)$ & $10(62.5)$ & $15(93.7)$ & $6(37.5)$ & $1(6.3)$ & $9(56.2)$ & $13(81.3)$ & $0(0)$ & - \\
\hline \multicolumn{10}{|c|}{ Haemophilus spp. $(n=3)$} \\
\hline$S$ & $3(100)$ & $1(33.3)$ & $0(0)$ & $2(66.7)$ & $3(100)$ & $2(66.7)$ & $0(0)$ & $3(100)$ & - \\
\hline$R$ & $0(0)$ & $2(66.7)$ & $3(100)$ & $1(33.3)$ & $0(0)$ & $1(33.3)$ & $3(100)$ & $0(0)$ & - \\
\hline \multicolumn{10}{|c|}{ Acinetobacter spp. $(n=5)$} \\
\hline$S$ & $5(100)$ & $5(100)$ & $0(0)$ & $3(60)$ & $5(100)$ & $3(60)$ & $0(0)$ & $5(100)$ & $3(60)$ \\
\hline$R$ & $0(0)$ & $0(0)$ & $5(100)$ & $2(40)$ & $0(0)$ & $2(40)$ & $5(100)$ & $0(0)$ & $2(40)$ \\
\hline \multicolumn{10}{|l|}{ Total $(N=46)$} \\
\hline$S$ & $43(93.5)$ & $26(56.5)$ & $4(8.7)$ & $31(67.4)$ & 45 (98) & $22(47.8)$ & $2(4.3)$ & $43(93.5)$ & - \\
\hline$I$ & $0(0)$ & $0(0)$ & $0(0)$ & $1(2.1)$ & $0(0)$ & $2(4.3)$ & $2(4.3)$ & $0(0)$ & - \\
\hline$R$ & $3(6.5)$ & $20(43.5)$ & $42(91.3)$ & $14(30.4)$ & $1((2.2)$ & $22(47.8)$ & $42(91.3)$ & $3(6.5)$ & - \\
\hline
\end{tabular}

Note. CIP, ciprofloxacin; COT, cotrimoxazole; CN, gentamicin; AMP, ampicillin; AMC, amoxicillin-clavulanic acid; TE, tetracycline; CRO. ceftriaxone; $C$, chloramphenicol; Pi, piperacillin; $S$, sensitivity; $I$, intermediate; $R$, resistance.

TABle 5: Multidrug-resistant patters of the bacterial isolates among blood culture-positive HIV/AIDS patients at Felege Hiwot Referral Hospital.

\begin{tabular}{|c|c|c|c|c|c|c|c|}
\hline \multirow{2}{*}{ Bacterial isolate } & \multicolumn{7}{|c|}{ Class of drugs } \\
\hline & $\mathrm{R} 0, N(\%)$ & $\mathrm{R} 1, N(\%)$ & $\mathrm{R} 2, N(\%)$ & $\mathrm{R} 3, N(\%)$ & $\mathrm{R} 4, N(\%)$ & $\geq \mathrm{R} 5(\%)$ & Overall MDR (\%) \\
\hline \multicolumn{8}{|l|}{ Gram-positive } \\
\hline S. aureus $(n=38)$ & $0(0)$ & $1(2.6)$ & $0(0)$ & $7(18.4)$ & $4(10.5)$ & $26(68.4)$ & $37(48)$ \\
\hline CoNS $(n=27)$ & $0(0)$ & $2(7.4)$ & $3(11.1)$ & $4(14.8)$ & $5(18.5)$ & $13(48.1)$ & $22(28.6)$ \\
\hline$S$ pneumoniae $(n=8)$ & $0(0)$ & $1(12.5)$ & $1(12.5)$ & $4(50)$ & $0(0)$ & $2(25)$ & $6(7.8)$ \\
\hline Enterococcus spp. $(n=4)$ & $0(0)$ & $1(25)$ & $0(0)$ & $1(25)$ & $1(25)$ & $1(25)$ & $3(3.9)$ \\
\hline Total $(n=77)$ & $0(0)$ & $5(6.5)$ & $4(5.2)$ & $16(20.8)$ & $10(13)$ & $42(54.5)$ & $68(88.3)$ \\
\hline \multicolumn{8}{|l|}{ Gram-negative } \\
\hline K pneumoniae $(n=19)$ & $2(10.5)$ & $0(0)$ & $6(31.5)$ & $4(21)$ & $2(10.5)$ & $5(26.3)$ & $11(23.9)$ \\
\hline K. rhinoscleromatis $(n=3)$ & $0(0)$ & $0(0)$ & $0(0)$ & $3(100)$ & $0(0)$ & $0(0)$ & $3(6.5)$ \\
\hline E aerogenes $(n=16)$ & $0(0)$ & $0(0)$ & $3(18.8)$ & $7(43.7)$ & $3(18.8)$ & $3(18.8)$ & $3(6.5)$ \\
\hline Haemophilus spp. $(n=3)$ & $0(0)$ & $1(33.3)$ & $2(66.7)$ & $0(0)$ & $0(0)$ & $0(0)$ & $0(0)$ \\
\hline Acinetobacter spp. $(n=5)$ & $0(0)$ & $3(60)$ & $1(20)$ & $1(20)$ & $0(0)$ & $0(0)$ & $1(2.2)$ \\
\hline Total $(n=46)$ & $2(4.3)$ & $4(8.7)$ & $12(28.2)$ & $15(32.6)$ & $5(11)$ & $8(17.3)$ & $28(60.8)$ \\
\hline Overall $(n=123)$ & $2(1.6)$ & $9(7.3)$ & $16(13)$ & $31(25.2)$ & $15(12.1)$ & $50(40.7)$ & $96(78)$ \\
\hline
\end{tabular}

Note. $R_{0}$, nonresistance; $R_{1}$, resistance for 1 antibiotic; $R_{2}$, resistance for 2 antibiotic; $R_{3}$, resistance for 3 antibiotics; $R_{4}$, resistance for 4 antibiotic; $R_{5}$, resistance for 5 and above.

percentage is slightly comparable to a study conducted in Gondar town, Ethiopia, in which $100 \%$ of Gram-negative and greater than $75 \%$ of Gram-positive bacteria were sensitive to ciprofloxacin [17]. Similarly, the study in Kampala, Uganda, among HIV-1 infected children showed that more than $80 \%$ of the bacterial isolates were susceptible to ciprofloxacin [23]. Another study from India indicated that among bacterial isolates, ceftriaxone and ciprofloxacin were effective with a very low resistance of $8 \%$ and $30.3 \%$, respectively [24].
In this study, a significant level of resistance to methicillin by S. aureus and coagulase-negative Staphylococci (CoNS) was observed, yielding $73.7 \%$ and $66.7 \%$ of methicillin-resistant $S$. aureus (MRSA) and methicillin-resistant coagulase-negative Staphylococci (MR-CoNS). This percentage of MRSA was significantly higher than findings reported in Bahir Dar, Ethiopia [25], and Italy, in which about $16.8 \%$ and $32 \%$, respectively, were MRSA among HIV/ AIDS patients [26]. 


\section{Limitations}

This study did not consider the antimicrobial resistance profile of some bacterial isolates such as Mycobacterium and anaerobic bacteria due to resource constraints, and also, it did not include parameters such as mortality rate and predisposing factors.

\section{Conclusions}

Based on our findings, we can conclude that the antimicrobial resistance patterns were high. Ciprofloxacin and vancomycin were found to be an active antibacterial agent for Gram-positive bacteria, while ceftriaxone, ciprofloxacin, and chloramphenicol were effective for Gram-negative bacteria. Most of the bacterial isolates in this study, $78 \%$ of them, showed MDR including cotrimoxazole, which is the most commonly prescribed prophylaxis agent in ART clinics. Blood culture tests should be considered for better management of bacterial bloodstream infections among HIV/AIDS patients. We recommend an additional study regarding mortality rate, loss of cost, and time due to bloodstream infection among HIV/AIDs patients. Besides, there should be continuous antimicrobial resistance surveillance in the study area on a large scale.

\section{Abbreviations}

AIDS: $\quad$ Acquired immunodeficiency syndrome

ART: Antiretroviral therapy

AMR: Antimicrobial resistance

BAP: $\quad$ Blood agar plate

CAP: $\quad$ Chocolate agar plate

CLSI: Clinical and Laboratory Standards Institute

CONS: Coagulase-negative Staphylococci

ECDC: European Centre for Disease Prevention and Control

HIV: Human immunodeficiency virus

MR- Methicillin-resistant coagulase-negative

CoNS: Staphylococci

MRSA: Methicillin-resistant Staphylococcus aureus.

\section{Data Availability}

The data used to support this study are available from the corresponding author upon request.

\section{Conflicts of Interest}

The authors declare that they have no conflicts of interest.

\section{Authors' Contributions}

M. J and Y. A designed the study, collected the data, entered into SPSS software, and cleaned up. T. D and T. B participated in the design of the study and the data collection process. All authors participated in the data analysis, interpretation, and development of the manuscript. All authors read and approved the final manuscript.

\section{Acknowledgments}

The authors acknowledge the Department of Medical Laboratory Sciences, Addis Ababa University, for supporting and encouragement throughout the study. The authors also would like to thank the Felege Hiwot Referral Hospital and Amhara Regional Laboratory for providing their laboratory facility and professionals. Finally, the authors would like to thank all the study participants. This study was funded by Addis Ababa University.

\section{References}

[1] A. G. W. L. Wasihun, S. A. Gebremariam, T. A. Dejene, A. L. Welderufael et al., "Bacteriological profile and antimicrobial susceptibility patterns of blood culture isolates among febrile patients in mekelle hospital, Northern Ethiopia," Springerplus, vol. 4, p. 314, 2015.

[2] L. K. Archibald, L. C. McDonald, O. Nwanyanwu et al., "A hospital-based prevalence survey of bloodstream infections in febrile patients in Malawi: implications for diagnosis and therapy," The Journal of Infectious Diseases, vol. 181, no. 4, pp. 1414-1420, 2000.

[3] A. O. Reller, O. Ojo Bola, and A. A. Olagbemi, "Prevalence and antibiotic resistance pattern of blood culture isolates from human immuno-deficiency virus (HIV) patients on highly active anti-retroviral therapy (HAART) in Nigeria," African Journal of Microbiology Research, vol. 9, no. 13, pp. 909-914, 2015.

[4] B.-L. Marie-Renée, D. Olivier, G. Bartlett et al., "Incidence and prevalence of opportunistic and other infections and the impact of antiretroviral therapy among HIV-infected children in low- and middle-income countries: a systematic review and meta-analysis," Clinical Infectious Diseases, vol. 62, no. 12, pp. 1586-1594, 2016.

[5] K. Gohel, J. Amit, S. Soni, S. Gang, R. Sabnis et al., "Bacteriological profile and drug resistance patterns of blood culture isolates in a tertiary care nephrourology teaching institute," BioMed Research International, vol. 5, 2014.

[6] K. Terfa Kitila, B. Dufera Tadesse, K. M. H. Tinsae et al., "Assessment of bacterial profile and antimicrobial resistance pattern of bacterial isolates from blood culture in Addis Ababa regional laboratory, Addis Ababa, Ethiopia," Clinical Microbiology, vol. 7, p. 2, 2018.

[7] Q. Yin, D. Yue, Y. Peng, Y. Liu, and L. Xiao, "Occurrence and distribution of antibiotic-resistant bacteria and transfer of resistance genes in Lake Taihu," Microbes and Environments, vol. 28, no. 4, pp. 479-486, 2013.

[8] R. Laxminarayan, A. Duse, C. Wattal et al., "Antibiotic resistance-the need for global solutions," The Lancet Infectious Diseases, vol. 13, no. 12, pp. 1057-1098, 2013.

[9] K. Le Doare, J. Bielicki, P. T. Heath, and M. Sharland, "Systematic review of antibiotic resistance rates among gramnegative Bacteria in children with Sepsis in resource-limited countries," Journal of the Pediatric Infectious Diseases Society, vol. 4, no. 1, pp. 11-20, 2015.

[10] C. Ntirenganya, C. M. Muvunyi, O. Manzi, and O. Ogbuagu, "High prevalence of antimicrobial resistance among common bacterial isolates in a tertiary healthcare facility in Rwanda," The American Journal of Tropical Medicine and Hygiene, vol. 92, no. 4, pp. 865-870, 2015.

[11] World Health Organization, Antimicrobial Resistance: Global Report on Surveillance, World Health Organization, Geneva, Switzerland, 2014. 
[12] K. S. WKaD, "Emergence of multidrug-resistant bacterial infection in HIV/AIDS cases," The Health, vol. 3, no. 2, pp. 49-52, 2012.

[13] CSA, Summary and Statistical Report of the 2007 Population and Housing Census, Addis Ababa, Ethiopia, 2008.

[14] S. A. Jolene Moore, "NHS Grampian. Felege Hiwot referral hospital, Ethiopia," 2016.

[15] WHO, Global Report on Surveillance. Antimicrobial Resistance, Global Report on Surveillance, World Health Organization, Geneva, Switzerland, 2014.

[16] Clinical and Laboratory Standards Institute, Performance Standards for Antimicrobial Disk Susceptibility Tests, Clinical and Laboratory Standards Institute Document M 100s, 26th edition, 2016.

[17] G. T. B. Alebachew, M. Endris, Y. Shiferaw, and B. Tessema, "Etiologic agents of bacterial sepsis and their antibiotic susceptibility patterns among patients living with human immunodeficiency virus at Gondar university teaching hospital, Northwest Ethiopia," BioMed Research International, vol. 2016, Article ID 5371875, 8 pages, 2016.

[18] A.-P. Magiorakos, A. Srinivasan, R. B. Carey et al., "Multidrug-resistant, extensively drug-resistant and pandrug-resistant bacteria: an international expert proposal for interim standard definitions for acquired resistance," Clinical Microbiology and Infection, vol. 18, no. 3, pp. 268-281, 2012.

[19] M. A. Gordon, "Salmonella infections in immunocompromised adults," Journal of Infection, vol. 56, no. 6, pp. 413-422, 2008.

[20] B. Liu, L. Zhang, R. Guo, J. Su, L. Li, and Y. Si, “Anti-infective treatment in HIV-infected patients during perioperative period," AIDS Research and Therapy, vol. 9, no. 1, p. 36, 2012.

[21] B. Afessa, I. Morales, and B. Weaver, "Bacteremia in hospitalized patients with human immunodeficiency virus," $B M C$ Infectious Diseases, vol. 1, p. 13, 2001.

[22] K. J. M. M. Maarwa, E. Konje, P. E. Alele, J. Kidola, and M. M. Mirambo, "Resistance to cotrimoxazole and other antimicrobials among isolates from HIV/AIDS and non-HIV/ AIDS patients at Bugando medical center, mwanza, Tanzania," AIDS Research and Treatment, vol. 2015, Article ID 103874, 2015.

[23] S. Patricia Rojas and H. Africa, "Drug resistance in the HIV-1infected paediatric population worldwide: a systematic review," Journal of Antimicrobial Chemotherapy, vol. 69, no. 8, pp. 2032-2042, 2014.

[24] A. A. S. Garg, J. Garg, R. K. Goyal, and M. R. Sen, "Bacteriological profile and antimicrobial resistance of blood culture isolates from a university hospital in India," Journal of Indian Academy of Clinical Medicine, vol. 8, no. 2, pp. 139-143, 2007.

[25] M. T. Z. Y. Lemma, B. Tulu, D. Mekonnen, and Z. Mekonnen, "Methicillin resistance Staphylococci aureus among HIV infected pediatric patients in Northwest Ethiopia: carriage rates and antibiotic Co-resistance profiles," PLoS One, vol. 10, no. 9, Article ID 0137254, 2015.

[26] M. Tumbarello, E. Tacconelli, R. Citton, T. Spanu, F. Leone et al., "Risk factors and predictors of mortality of methicillinresistant Staphylococcus aureus (MRSA) bacteraemia in HIVinfected patients," Journal of Antimicrobial Chemotherapy, vol. 50, no. 3, pp. 375-382, 2002. 